\section{Recent patents related to aquaporins}

Aquaporins are a class of membrane proteins that facilitate water transport across the plasma membranes of cells and are involved in epithelial fluid secretion, cell migration, brain oedema and adipocyte metabolism. As Verkman and colleagues discuss on page 259, these channels hold potential for the treatment of several disorders, including oedema, cancer, obesity and brain injury, but identifying modulators of these channels has posed a considerable challenge.

Here in TABLE 1 we highlight selected patent applications published in the past 2 years related to aquaporins. Data were researched using the Espacenet database.

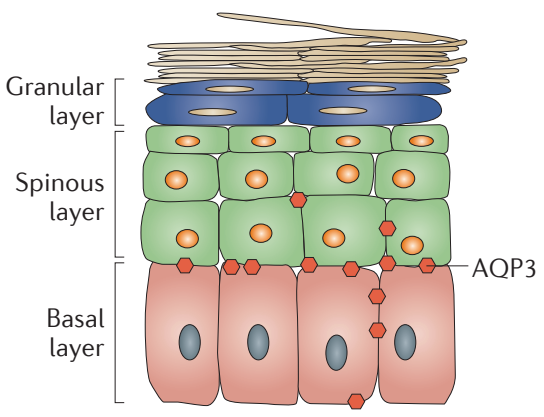

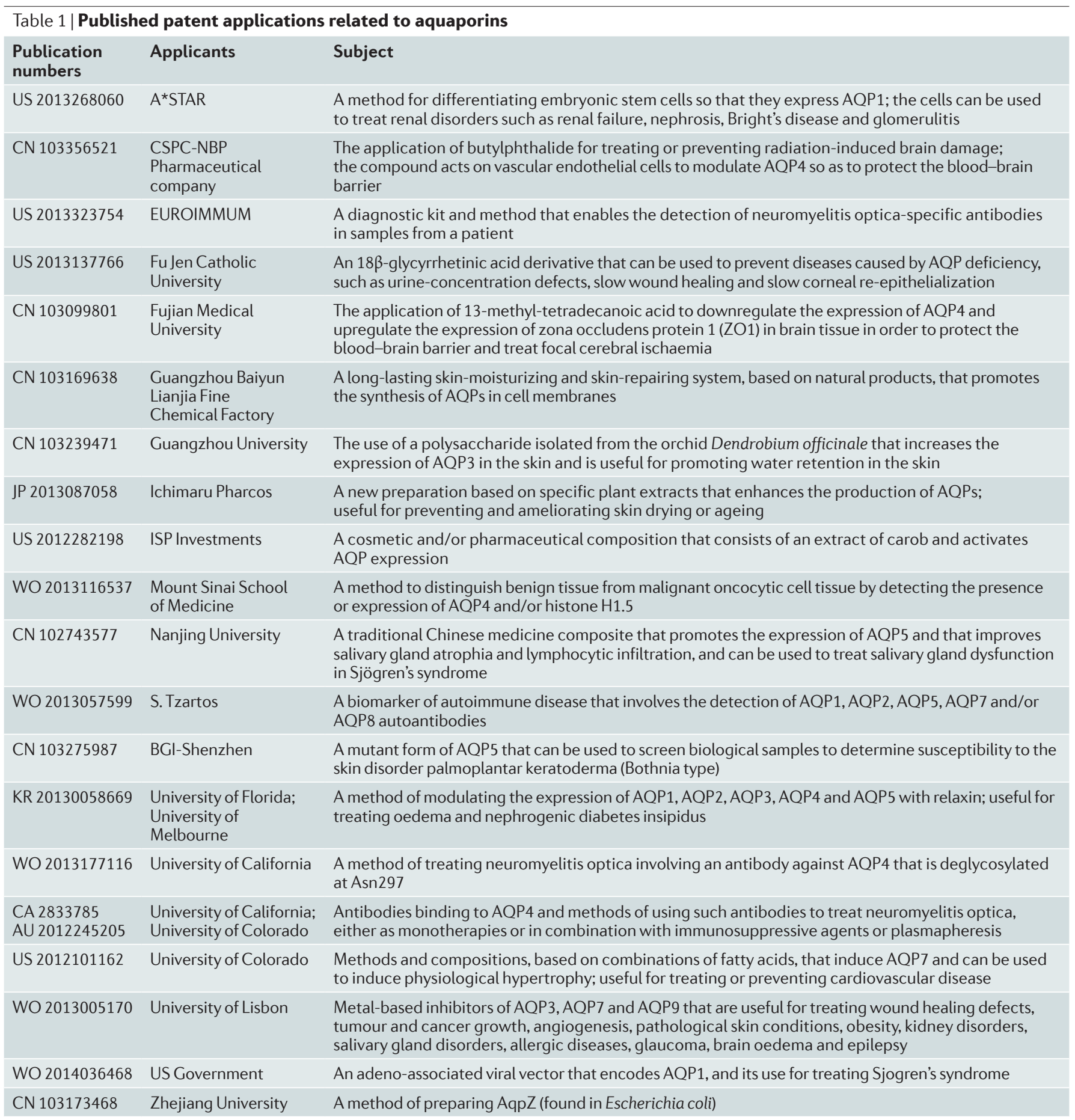

AQP, aquaporin; A*STAR, Agency for Science, Technology and Research; BGI, Beijing Genomics Institute. 